\title{
Further study of differential conditioning in the planarian'
}

Twelve planaria were cut in half and regenerated. One member of each regenerated pair received 30 paired trials of light plus shock and 30 unpaired presentations of a vibrator each day for 5 days while the other member of each pair received vibrator plus shock and light unpaired. On the sixth day all 24 animals were given 30 trials of light and 30 of vibrator, both unpaired with shock. During the five days of ac quisition the vibrator-plus-shock group showed significant differential responding while the light-plus-shock group did not. In extinction both groups made more responses to the vibrator than to the light, regardless of previous pairing with shock. Since the pilot study had shown the vibrator and light to have equal unconditioned response-evocation strength, the difference could not be attributed to a simple dynamogenic process. It could not be concluded unequivocally that differential conditioning in the planarian is possible.

An earlier study (Kimmel \& Harrell, 1964) of differential classical conditioning in the planarian had shown differentiation between reinforced and nonreinforced stimuli only when a vibrator was paired with shock but not when a light was paired with shock. The possibility existed that the vibrator and light differed in response-evocation strength even before training, however. Thus, it was decided to repeat the study on a larger scale with equipotential stimuli and to include an extinction session following training. Intersubject variations were controlled by using regenerated halves of the same animal as their own controls.

Method

Twelve planaria, Dugesia tigrina, were cut in half and given 14 days to regenerate in a dark room. Head sections were randomly assigned in equal numbers to the light reinforced ( $(\mathrm{L}+)$ and the vibrator $(\mathrm{V}+)$ groups and their corresponding tail sections to the opposite groups. $^{2}$ On five successive training days each $\mathrm{S}$ received a randomized sequence of 30 lights and vibrators (the appropriate CS paired with shock and the other one unpaired) in a Lucite conditioning trough. The intensities of the light and vibration (a $40 \mathrm{w}$ frosted bulb 6 in. above the trough and a telephone buzzer attached to the side of the trough, respectively) were selected in a pilot study of 10 additional Ss, so that each produced about three-four unconditioned responses in 60 trials. The duration of each CS was 3 sec., the shock sharing the last $1 \mathrm{sec}$, with the CS. The ITI was always at least $15 \mathrm{sec}$. and a trial was run only when the $S$ was swimming in the longitudinal plane of the
H. D. KIMMEL, OHIO UNIVERSITY VIRGINIA L, HARRELL, UNIVERSITY OF FLORIDA

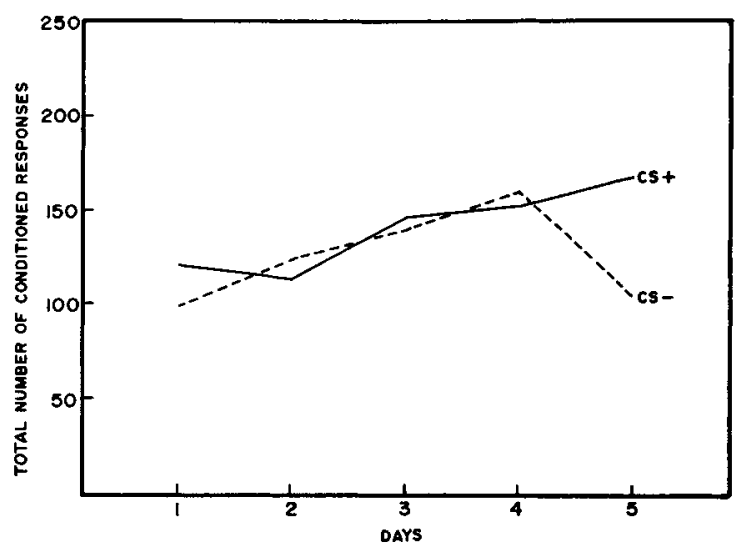

Fig. 1. Total number of CRs to the light and vibrator during training in the $\mathbf{L}+$ group.

trough towards the cathode. Following training 60 extinction trials were given, 30 with each $\mathrm{CS}$, in a randomized sequence. Aside from changes in the stimuli, the equipment was the same as used by Baxter \& Kimmel (1963) and Kimmel \& Harrell (1964).

Results

A CR was defined as a cephalic turning, curling, or contraction during the first 2 sec. of the light or vibrator. Figure 1 shows the total number of CRs made to each stimulus by the $L+$ group on each of five training days and Fig. 2 shows the same data for the V+ group. The overall difference between $S+$ and $S-$ was significant $(p<.05$, Wilcoxen test) during training only in the V+ group but not in the L+ group. Inspection of

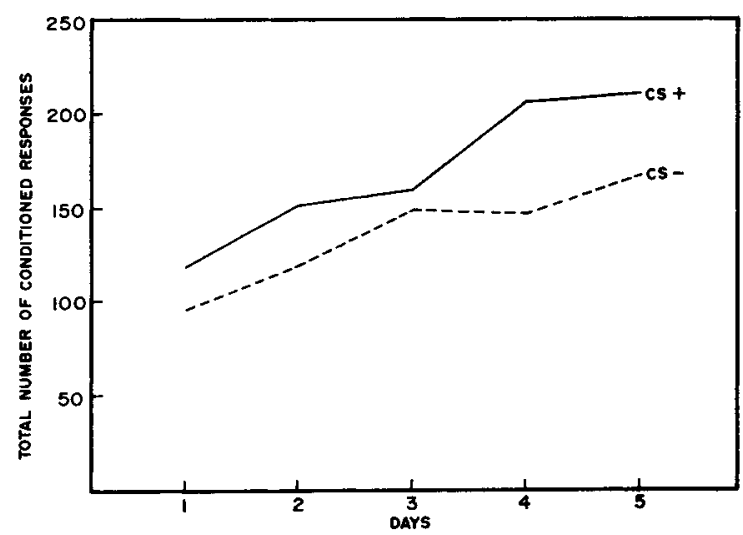

Fig. 2. Total number of CRs to the light and vibrator during training in the $\mathrm{V}+$ group. 

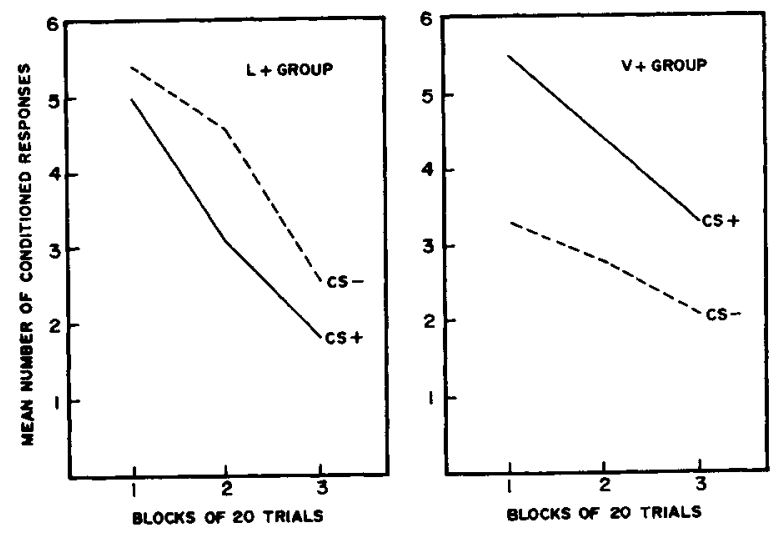

Fig. 3. Mean number of CRs to the light and vibrator during extinction in the $L_{+}$and $V_{+}$groups.

the two figures indicates that responding to the light was about the same in both groups, regardless of pairing with the shock, while the vibrator appears to have been influenced by pairing with the shock.

Figure 3 shows the mean number of responses to the light and vibration in the $\mathrm{L}+$ and $\mathrm{V}+$ groups during extinction. While the difference in responding to S+ and S- was not significant in either group, it is clear that the vibrator produced more responses in both groups, regardless of the previous reinforcement history. Discussion

The results of this study are in complete consonance with those reported earlier by Kimmel \& Harrell (1964). It was felt that matching the intensities of the two CSs would clear up the possibility that the previous superiority of the vibrator and lack of conditioning with the light resulted from intensity differences. Even with equivalent initial response-evocation strengths, however, differential responding was achieved only in the $\mathrm{V}+$ group but not in the L+ group. Furthermore, neither group showed significant differential responding in extinction, although the vibrator produced more responses than the light in both groups.

With the problem of initial intensity differences more or less eliminated from consideration, the next most likely explanation of the differential responding in the $\mathrm{V}+$ but not the $\mathrm{L}+$ groups would seem to be the greater physical similarity between the vibrator and the shock. Perhaps the apparent differential conditioning in the $\mathrm{V}+$ group was due to generalization from the shock to the vibrator? If this, in fact, were the case, one could hardly argue that "true" differential conditioning was obtained in this study. It does not seem appropriate at present to conclude that the planarian shows this type of conditioning, although it is possible that a longer training session might permit differentiation to occur.

\section{References}

Baxter, R., \& Kimmel, H. D. Conditioning and extinction in the planarian. Amer. J. Psychol., 1963, 56, 597-599.

Kimmel, H. D., \& Harrell, y. L. Differential conditioning in the planarian. Psychon. Sci., 1964, 1, 227-228.

\section{Notes}

1. Based on the second author's M. A. Thesis at the University of Florida.

2. One of the L+ Ss stopped gliding completely on Day 2 and had to be dropped from the experiment. Thus $\mathrm{L}+\mathrm{N}=11$ and $\mathrm{V}+\mathrm{N}=12$. 